\title{
Apoptosis in hypertensive heart disease: a clinical approach Arantxa González ${ }^{\mathrm{a}}$, Susana Ravassa ${ }^{\mathrm{a}}$, Begoña López ${ }^{\mathrm{a}}$, Iñigo Loperena ${ }^{\mathrm{a}}$, Ramón Querejeta ${ }^{\mathrm{b}}$ and Javier Díez ${ }^{\mathrm{a}, \mathrm{c}}$
}

\begin{abstract}
Purpose of review
It is widely accepted that there are two principal forms of cell death, namely, necrosis and apoptosis. According to the classical view, necrosis is the major mechanism of cardiomyocyte death in cardiac diseases.

\section{Recent developments}

In the past few years observations have been made showing that cardiomyocyte apoptosis occurs in diverse conditions including hypertensive heart disease, and that apoptosis may be a contributing cause of loss and functional abnormalities of cardiomyocytes in this condition.

\section{Summary}

This review will summarize recent evidence demonstrating the potential contribution of cardiomyocyte apoptosis to heart failure in hypertensive patients. In addition, some strategies aimed to detect and prevent apoptosis of cardiomyocytes will be considered.
\end{abstract}

\section{Keywords}

apoptosis, cardiomyocytes, heart failure, hypertensive heart disease

Curr Opin Cardiol 21:288-294. (C) 2006 Lippincott Williams \& Wilkins.

aDivision of Cardiovascular Sciences, Centre for Applied Medical Research, School

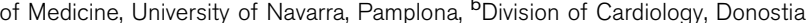

University Hospital, San Sebastián and ${ }^{\mathrm{C}}$ Department of Cardiology and

Cardiovascular Surgery, University Clinic, School of Medicine, University of Navarra, Pamplona, Spain

Correspondence to Dr Javier Díez, Área de Ciencias Cardiovasculares, Centro de Investigación Médica Aplicada, Avda. Pío XII, 55, 31008 Pamplona, Spain Tel: +34 948 194700; fax: +34948 194716; e-mail: jadimar@unav.es

Current Opinion in Cardiology 2006, 21:288-294

\section{Abbreviations}

ATP adenosine triphosphate

HHD hypertensive heart disease

IGF-I insulin-like growth factor-I

SHR spontaneously hypertensive rats

(C) 2006 Lippincott Williams \& Wilkins 0268-4705
Introduction

It has been classically accepted that adult cardiomyocytes are not capable of proliferation and, thus, are resistant to developing apoptosis. Thus, the existence of a balance between apoptotic cell death and cell regeneration in the heart has been denied until recently. In the past decade, however, observations have been made showing that cardiomyocyte apoptosis occurs in diverse conditions $\left[1^{\bullet \bullet}, 2^{\bullet \bullet}\right]$. One of these conditions is hypertensive heart disease (HHD), here defined by the presence of a greater than normal left ventricular mass in the absence of a cause other than arterial hypertension and by the development of complex changes in myocardial composition that are responsible for the structural remodeling of the myocardium (Table 1). One of these changes is a diminished number of cardiomyocytes due to enhanced cell death, including apoptotic cell death. In fact, cardiomyocyte apoptosis has been shown to be abnormally stimulated in the hypertrophied left ventricle of patients with essential hypertension, no angiographic evidence of coronary artery disease and normal cardiac function [3,4]. In addition, recent findings from our laboratory show that cardiomyocyte apoptosis is increased in patients with HHD and chronic heart failure compared with hypertensive patients with HHD and normal cardiac function [ $5^{\bullet \bullet}$ ] (Fig. 1). Thus, it seems that cardiomyocyte apoptosis precedes the impairment in ventricular function and its exacerbation accompanies the development of heart failure in patients with HHD.

\section{Mechanistic links between cardiomyocyte apoptosis and heart failure in hypertensives}

Arterial hypertension affects the myocardium at two different stages [6]. In both humans and animal models, pressure overload is characterized by a period of compensation in which left ventricular concentric hypertrophy normalizes systolic wall stress and contractile function is preserved. The period of adaptation, which may last for weeks in rodents and months to years in humans, is inexorably followed by a transition to cardiac failure. This transition is characterized by impaired survival, the onset of chamber dilatation with the failure of further concentric hypertrophic growth to normalize load, and progressive contractile dysfunction. A number of observations suggest that beside changes in the composition of motor unit and cytoskeleton of cardiomyocytes, the transition from hypertrophy to failure relates mainly to alterations in the histological composition of the 
Table 1 Main histological components of hypertensive myocardial remodeling

Cellular changes

Hypertrophy of cardiomyocytes

Decreased number of cardiomyocytes

Increased number of fibroblasts

Phenotypic differentiation of fibroblasts into myofibroblasts

Presence of abundant inflammatory cells and mast cells

Noncellular changes

Interstitial and perivascular fibrosis

Wall thickening of intramyocardial vessels

Reduced number of capillaries

myocardium as a result of both alterations in the metabolism of extracellular matrix (namely, fibrillar collagen) and cardiomyocyte loss due to multiple mechanisms of death (including apoptosis). Beside reduction in the number of cardiomyocytes, apoptosis may contribute to heart failure through additional pathways $\left[7^{\bullet \bullet}, 8^{\bullet \bullet}\right]$ (Fig. 2).

\section{Apoptosis and structural and geometric remodeling}

It has been suggested that alterations of the collagen framework in the myocardium may play an important role in the genesis of diastolic dysfunction of hypertensive origin $\left[9^{\bullet}\right]$. This has been supported by the finding that fibrillar collagen deposition in the cardiac interstitium of hypertensive patients increases left ventricular chamber stiffness and compromises left ventricular filling during diastole [10]. The problem concerns whether this type of interstitial alteration occurs through activation of fibroblasts via humoral or mechanical factors in the absence of cardiomyocyte loss, or whether cell death is required for the stimulation of the growth response of the noncardiomyocyte compartment of the myocardium. The observation that fibrosis is associated with cell loss in the left ventricle of hypertensive patients [11] and patients with aortic stenosis [12] raises questions about the mechanism responsible for the modification of the interstitium with accumulation of fibrillar collagen. As proposed by Anversa $e t$ al. [13], death of individual cardiomyocytes may stimulate discrete healing processes contributing to the expansion of the interstitium. This proposal is further supported by the experimental finding that failing hearts from spontaneously hypertensive rats (SHR) present colocalization of collagen $\alpha_{1}$ type I gene expression to areas of focal cardiomyocyte degeneration [14], suggesting that cardiomyocyte loss is associated with collagen type I production and focal scar formation in these animals during the transition from hypertrophy to failure.

Besides histologic remodeling of the myocardium, cardiomyocyte apoptosis may also contribute to geometric remodeling of the left ventricular chamber. In fact, severe cardiomyocyte apoptosis may lead to side-to-side slippage of cells, mural thinning and chamber dilatation. Thus, wall restructuring secondary to severe cardiomyocyte apoptosis may create an irreversible state of the myocardium, conditioning progressive dilatation and the continuous deterioration of cardiac hemodynamics and ventricular performance with time $\left[15^{\bullet \bullet}\right]$.

\section{Apoptosis and energy production}

It is known that energy metabolism is deranged in the human hypertensive and failing myocardium. Although recent findings suggest that altered myocardial fatty acid metabolism may account for this abnormality in hypertensive patients [16], the possibility also exists that mitochondrial production of adenosine triphosphate (ATP) is diminished in the hypertensive myocardium [17]. In this setting it is interesting to consider that apoptosis is associated with loss of cytochrome $c$ from

Figure 1 Cardiomyocyte (CM) apoptosis (left panel) and density (right panel) in patients with hypertensive heart disease and normal cardiac function (non-heart failure) or clinical manifestations of heart failure (HF)

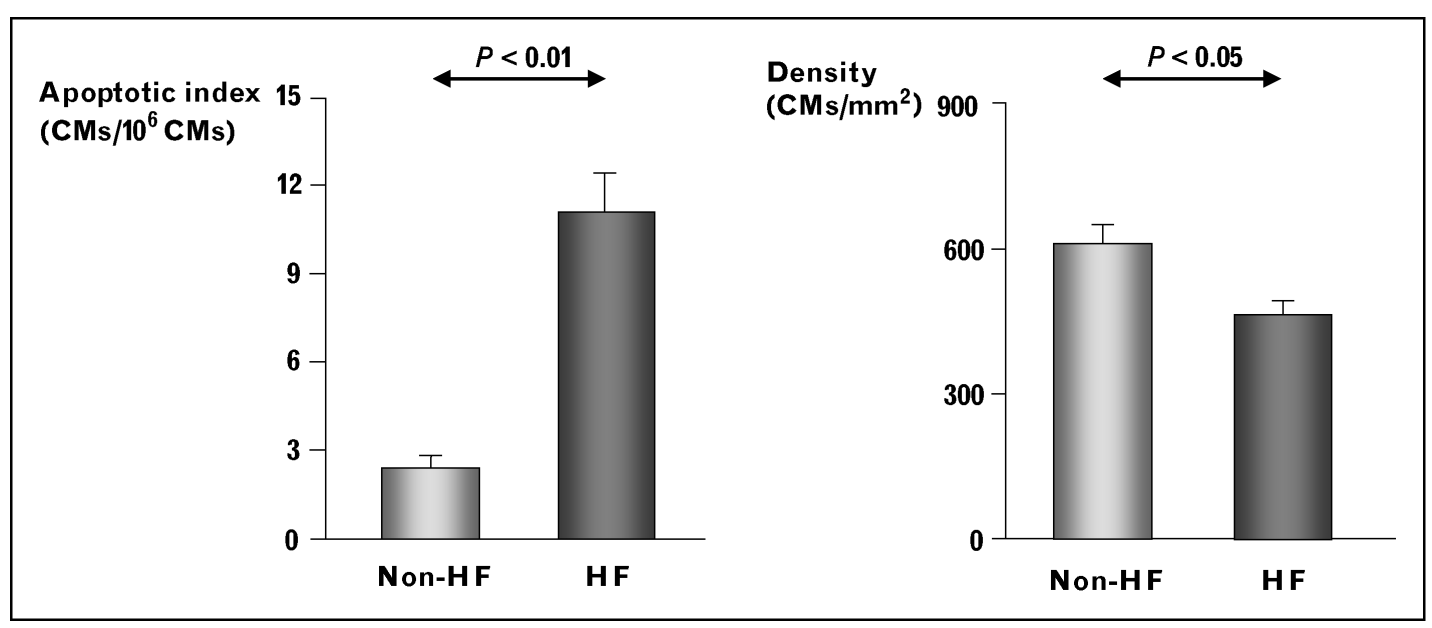


Figure 2 Pathways to heart failure activated by increased cardiomyocyte apoptosis in hypertensive heart disease

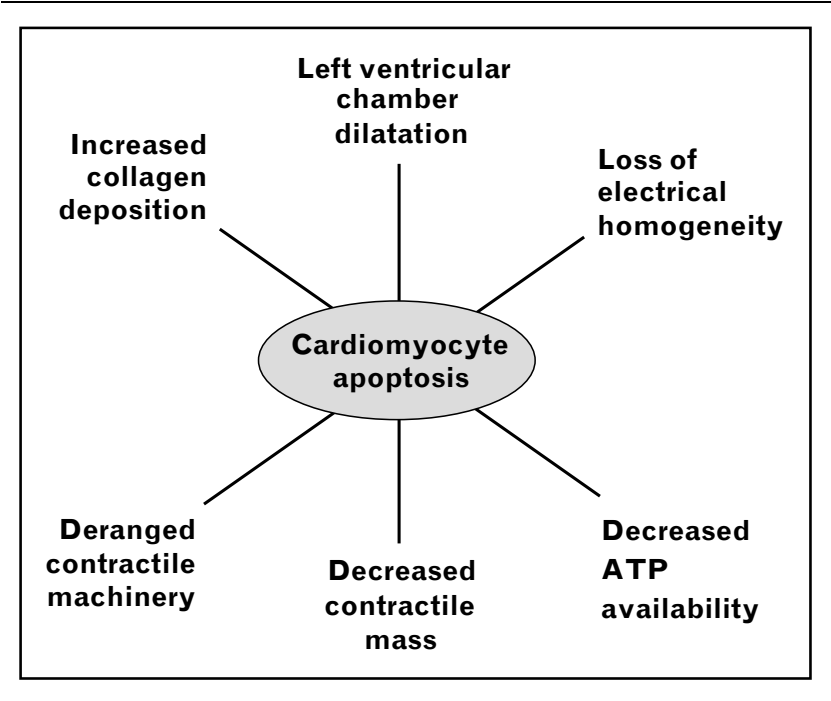

the mitochondria and this may halt oxidative phosphorylation and production of ATP, namely in subsarcolemmal mitochondria [18]. Nevertheless, as apoptosis is an energy-requiring process, in conditions of severely diminished ATP availability the mode of cell death would switch to necrosis. One might then predict that apoptotic stimuli do not necessarily induce complete loss of cytochrome $c$ from the mitochondria. Thus, the contribution of the mitochondrial pathway of apoptosis to the energy balance of the hypertensive myocardium remains to be clarified.

\section{Apoptosis and contractile mass}

As previously mentioned, increased cardiomyocyte apoptosis and diminished cardiomyocyte number have been found in the failing hearts of hypertensive patients [ $\left.5^{\bullet \bullet}\right]$. This has been confirmed at the experimental level in SHR with heart failure [19]. Thus, apoptosis may be one of the mechanisms involved in the loss of contractile mass and function in HHD. At present, however, there is no information concerning the magnitude of cell loss required to depress cardiac contractility in the hypertrophied human heart when cell death occurs. Although most studies examining apoptosis in the end-stage heart report variable but low apoptosis rates $(0.1-0.5 \%$ of cardiomyocytes), conservative assumptions about the duration of apoptosis and the constancy of the implied rate of cell death suggest that apoptosis could result in the loss of up to $5-10 \%$ of the myocardium per year [20]. Although the progressive nature and poor survival in latestage heart failure may well be consistent with such a substantial loss of cardiomyocytes, it means that the heart should rapidly disappear. This contention, however, does not consider that the adult heart possesses a stem cell compartment that can regenerate cardiomocytes and coronary vessels $\left[21^{\bullet \bullet}\right]$. This does not imply that cell replication compensates for the extent of apoptotic loss in the diseased myocardium, but allows the hypothesis that inadequate cardiomyocyte division may be a critical event in the evolution of the pathological heart to heart failure [22].

\section{Apoptosis and contractility}

Impaired myocardial contractile function may reflect not only a decrease in the number of viable, fully functional cardiomyocytes, but also a decrement in the function of viable cardiomyocytes, or a combination of these mechanisms. It is well known that apoptosis is associated with activation of caspases that mediate the cleavage of vital and structural proteins. Caspase- 3 cleaved cardiomyocyte myofibrillar proteins resulting in an impaired force/Ca ${ }^{2+}$ relationship and myofibrillar ATPase activity have been reported [23]. In addition, it has been demonstrated that caspase activation is associated with cleavage of myofilaments, disruption of sarcomeric structure and reduction in contractile force of failing cardiomyocytes, and that blockade of caspase activation improves contractility in failing myocardium [24 $4^{\bullet}$. As apoptosis may not be complete in cardiomyocytes, allowing the cells to persist for a prolonged period within the myocardium, the detrimental effects of caspase activation on systolic function should not be underestimated. This possibility is particularly relevant taking into account that an intense expression of the active form of caspase- 3 has been reported in cardiomyocytes from patients with HHD and heart failure [25].

\section{Apoptosis and electrical activity}

HHD is associated with increased incidence of both ventricular arrhythmias and sudden cardiac death, namely when heart failure is present [26]. It has been proposed that cardiomyocyte apoptosis may contribute to the development of arrhythmias in the failing heart through two ways [27]. First, in the progress of dying, a cardiomyocyte passes through phases of increased excitability or becomes automatic, at least until it is dead. Second, from a random grouping of several such dead cells, the process of normal activation in that area of heart muscle must be deranged and redirected in a way that would provide a suitable anatomical substrate for reentrant arrhythmias.

\section{Clinical recognition of cardiac apoptosis}

Thus far, methods by which to measure apoptosis were designed for in-vitro analysis. Most of these methods require tissue biopsy specimens to be obtained from the patient to retrieve information of apoptosis in situ. This limits the application of these apoptosis detection methods in clinical practice. A noninvasive detection method by which to visualize apoptosis would be ideal to assess its role in disease and to measure treatment outcome in diseases such as HHD. 


\section{Imaging studies}

Some molecular changes characteristic of apoptosis have been proposed as potential markers to be used in imaging techniques (e.g. nuclear magnetic resonance and radionuclide imaging) to detect previously inaccesible and unrecognized biological phenomena in living cells and tissues undergoing apoptosis [28,29].

For instance, apoptosis activates mechanisms which cause the translocation of phosphatidylserine from the internal to the external leaflet of the plasma membrane [30]. Annexin V is a phospholipid binding protein, which, in the presence of $\mathrm{Ca}^{2+}$ ions, specifically and reversibly interacts with the phosphoserine headgroup of phosphatidylserine in the apoptotic cell [31] (Fig. 3). This property has been the driving force for the research of annexin $\mathrm{V}$ conjugated with a detectable marker, such as biotin, a fluorochrome, or a radioligand, as a probe to measure apoptosis in vitro and in vivo in animals and patients [32]. Technetium-99 m labeled annexin $\mathrm{V}$ has been successfully used for noninvasive $\gamma$-imaging of cardiac apoptosis after acute myocardial infarction, acute myocardial ischemia, acute cardiac allograft rejection and malignant intracardiac tumors [33].

\section{Biochemical assessment}

Besides imaging studies, determination of circulating annexin $\mathrm{V}$ may be also useful for the biochemical monitoring of the apoptotic process. In this respect, it has been reported in humans that plasma levels of annexin $\mathrm{V}$ determined by means of ELISA are increased 8 -fold in the early phase of acute myocardial infarction, and immediately decrease after the onset of pain [34]. Other circulating markers of apoptosis are currently under investigation. For instance, it has been recently

Figure 3 The arrow signals an annexin V-positive cardiomyocyte and the arrowhead signals a control nonstained cardiomyocyte

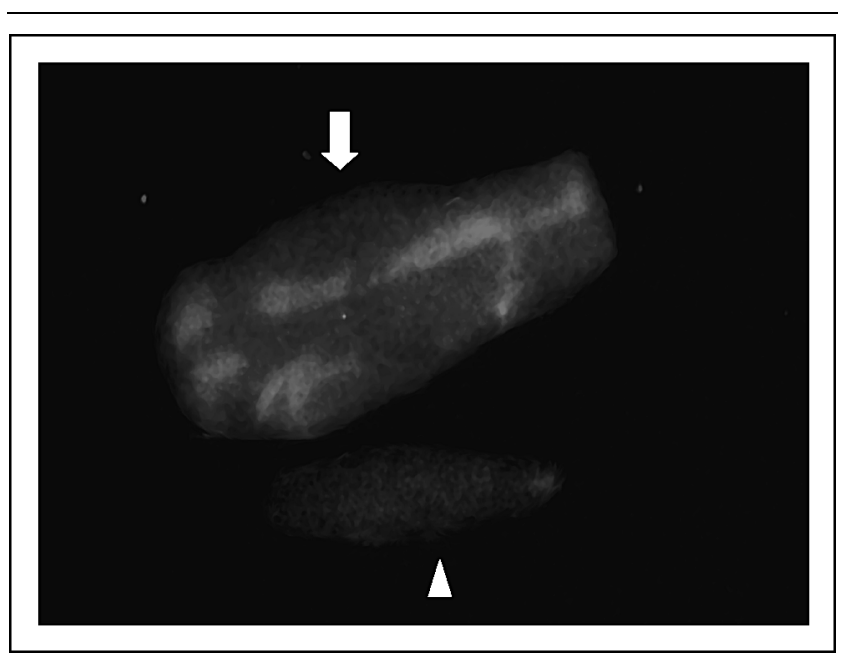

Annexin $\mathrm{V}$ staining was detected with tetramethylrhodamine-conjugated extravidine. reported that during apoptosis cytochrome $c$ not only translocates into the cytosol but is secreted to the extracellular medium [35]. Thus, its potential role as a serum marker of cardiac apoptosis in chronic cardiomyopathies, including HHD, remains to be investigated.

\section{Therapeutic modulation of cardiac apoptosis}

It has been postulated that the inhibition of cardiomyocyte apoptosis could prevent or slow cardiac failure progression, thus opening new strategies in the treatment of cardiac diseases $\left[36^{\bullet \bullet}, 37^{\bullet \bullet}\right]$. Cardiomyocyte apoptosis may be inhibited by suppressing the systemic and local factors that trigger the process, by directly blunting the intracellular apoptotic pathways or by inducing the survival pathways.

\section{Strategies aimed to blunt triggers of the apoptotic process}

The main systemic trigger of apoptosis to be considered in HHD is arterial hypertension itself. The in-vivo effects of antihypertensive drugs on cardiac apoptosis in SHR have been reviewed elsewhere [38]. Collectively, the available findings suggest that the ability of antihypertensive drugs to inhibit cardiac apoptosis is independent of their antihypertensive efficacy, but can be related to their capacity to interfere with the pro-apoptotic actions of humoral factors. This is further supported by clinical findings showing that despite an identical antihypertensive efficacy, the angiotensin II type 1 receptor antagonist losartan, but not the $\mathrm{Ca}^{2+}$ channel blocker amlodipine, reduced cardiomyocyte apoptosis in patients with HHD after 1 year of treatment [4].

Oxidant stress is an important local factor inducing cardiomyocyte apoptosis. Thus, antioxidant therapy, whether through administration of additional antioxidants or boosting innate antioxidant mechanisms, has been shown to be a viable approach in reducing cardiac apoptosis in experimental conditions $\left[39^{\bullet}, 40^{\bullet}\right]$. In the clinical setting, agents with antioxidant properties such as carvedilol or those that promote antioxidant activity, such as propranolol, are already used to treat patients with heart failure [41]. One ongoing study is testing the effect of oxypurinol, the major metabolite of the xanthine oxidase inhibitor allopurinol, in patients with heart failure [42]. The rationale for this study is that oxypurinol has been reported to substantially improve the cardiac structure and function in SHR with heart failure [43] and that xanthine oxidase has been identified as a source of reactive oxygen species in patients with heart failure [44].

\section{Strategies aimed to interrupt the apoptotic process}

The past decade has witnessed tremendous progress in the knowledge of the molecular mechanisms that regulate and execute apoptosis at the intracellular level. Consequently, apoptosis regulators and executors have 
Table 2 Potential molecular targets of the apoptotic machinery

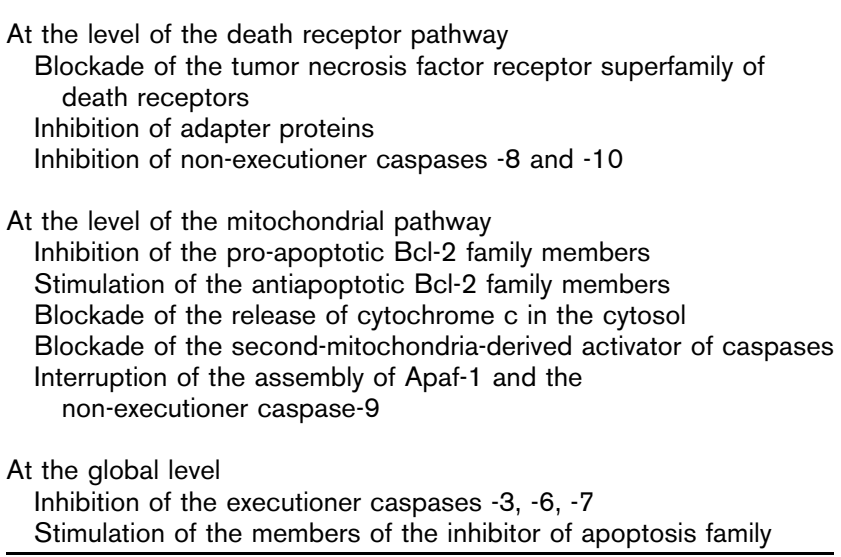

emerged as key targets for the design of therapeutic strategies aimed at interrupting cellular death decisions $\left[36^{\bullet \bullet}, 45^{\bullet \bullet}\right]$ (Table 2).

For instance, increased survivin (a member of the inhibitor of apoptosis [IAP] family) myocardial expression in aging SHR with heart failure is associated with reduced cardiomyocyte apoptosis and more favorable echocardiographic indexes of left ventricular remodeling and dysfunction $\left[46^{\bullet}\right]$. On the other hand, overexpression of human antiapoptotic protein $\mathrm{Bcl}-2$ decreases cardiac apoptosis and improves cardiac function in transgenic mice after ischemia/reperfusion [47]. By contrast, targeted deletion of pro-apoptotic members of the Bcl-2 protein family in a mouse model of cardiac ischemia/ reperfusion attenuated both apoptosis and necrosis [ $\left.{ }^{\bullet} 8^{\bullet}\right]$. Similar effects have been reported with low molecular weight caspase inhibitors $\left[4^{\bullet}\right]$. Interestingly, some of these inhibitory compounds were effective not only in reducing cardiomyocyte apoptosis but also in improving cardiac function and delaying heart failure development [50].
Other studies are focusing on the stimulation of the ubiquitin-proteasome system that contributes to regulation of apoptosis through the degradation of apoptosis-regulatory proteins. This is based on the recent observation that depression of proteasome activities in pressure-overloaded hearts of mice contributes to cardiac dysfunction resulting from cardiomyocyte apoptosis through accumulation of pro-apoptotic proteins by impaired degradation $\left[51^{\bullet \bullet}\right]$.

\section{Strategies aimed to stimulate cellular survival mechanisms}

An alternative strategy to prevent cardiomyocyte apoptosis is the improvement of cellular survival mechanisms $\left[52^{\bullet \bullet}\right]$. For instance, cardiotrophin-1 has been shown to protect cardiomyocytes against angiotensin II and oxidative stress-induced cell death via gp130/LIFR and by means of the PI3K/Akt and p42/44 MAPK intracellular cascades [53]. These aspects can be of particular relevance taking into account that molecular and functional alterations of the gp130 survival pathway have been described recently in failing human hearts [54].

Another important cardiomyocyte survival factor is insulin-like growth factor-I (IGF-I). In fact, in-vitro and in-vivo studies have shown that IGF-I decreases experimentally induced cardiomyocyte apoptosis through the activation of signaling pathways similar to those activated by cardiotrophin- 1 [5 $\left.^{\circ}\right]$. Interestingly, in clinical studies with patients with heart failure, IGF-I levels are low and correlate with the severity of left ventricular systolic dysfunction [56].

\section{Novel therapeutic approaches}

Several new treatment strategies are currently being explored in chronic heart failure, which, among other effects, have been shown to decrease cardiomyocyte apoptosis either clinically or experimentally. These

\section{Figure 4 Strategies to preserve the population of functional cardiomyocytes in hypertensive heart disease}

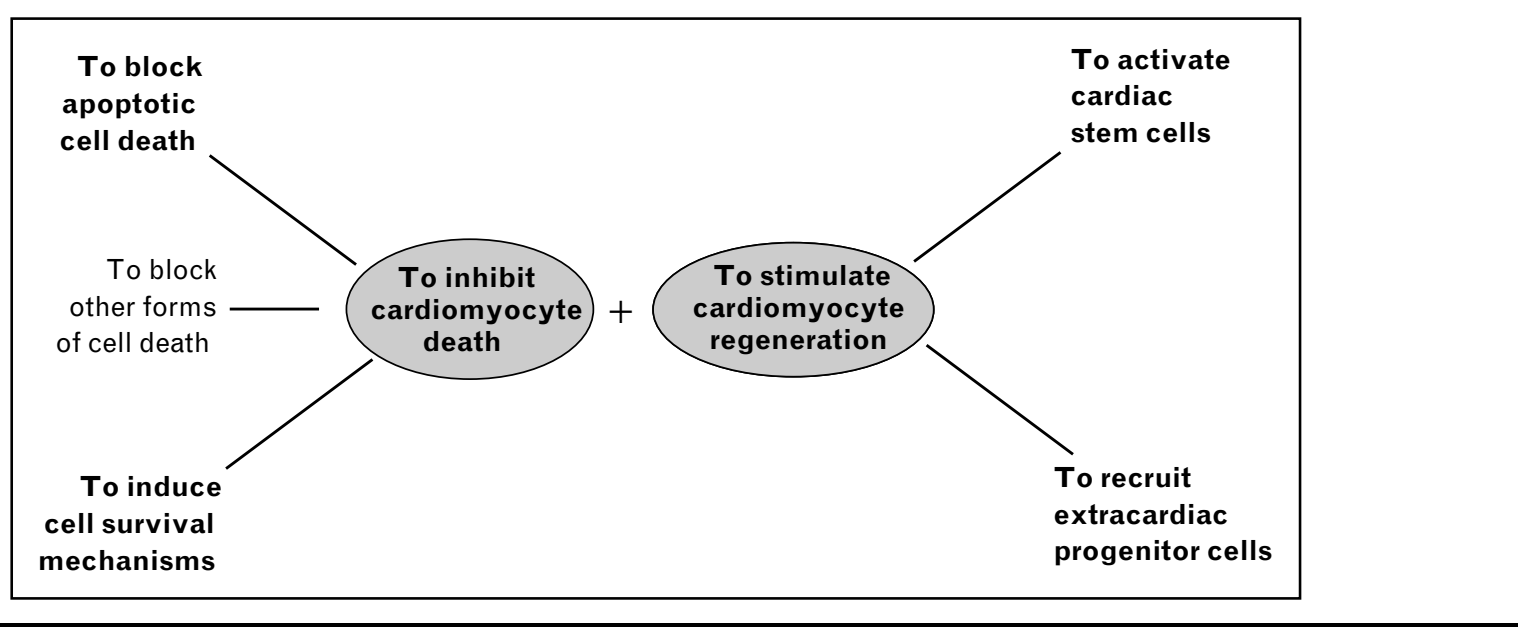


include medical therapy with mineralocorticoid receptor antagonists [57], calcium-sensitizing agents [58], statins [59] and erythropoietin [60], and device therapy aimed to induce cardiac resynchronization $\left[61^{\circ}\right]$.

\section{Conclusion}

Numerous hypotheses have been considered to explain the fundamental mechanism(s) for the development of heart failure in patients with HHD. Cardiomyocyte loss and dysfunction due to apoptosis is now being considered as one of the determinants of the maladaptive processes implicated in the transition from left ventricular hypertrophy to heart failure in hypertensive patients. Much work is being carried out regarding the mechanisms and the impact of cardiomyocyte apoptosis on the hypertensive myocardium but several methodological and conceptual issues still remain unsolved. Clarification of these is extremely urgent if one considers that the development of noninvasive tools for the monitoring of cardiac apoptosis and pharmacological strategies aimed to inhibit and/ or counteract the apoptotic process could be of particular relevance to protect the cardiomyocyte and prevent the progression to heart failure in patients with HHD (Fig. 4).

\section{References and recommended reading}

Papers of particular interest, published within the annual period of review, have been highlighted as:

- of special interest

$\bullet \quad$ of outstanding interest

Additional references related to this topic can also be found in the Current

World Literature section in this issue (pp. 408-409).

1 Regula KM, Kirshenbaum LA. Apoptosis of ventricular myocytes: a means to -• an end. J Mol Cell Cardiol 2005; 38:3-13.

Extensive review about apoptosis mechanisms in the context of adult cardiac muscle.

2 Foo RS, Mani K, Kitsis RN. Death begets failure in the heart. J Clin Invest -. $2005 ; 115: 565-571$.

This article reviews cardiomyocyte apoptosis in failing human hearts and its therapeutic implications.

3 Yamamoto S, Sawada K, Shimomura H, et al. On the nature of cell death during remodeling of hypertrophied human myocardium. J Mol Cell Cardiol 2000; 32:161-175

4 González A, López B, Ravassa S, et al. Stimulation of cardiac apoptosis in essential hypertension: potential role of angiotensin II. Hypertension 2002; 39:75-80.

5 Goikoetxea MJ, Beaumont J, González A, et al. Altered cardiac expresion of

- peroxisome proliferator-activated receptor alpha isoforms in patients with hypertensive heart disease. Cardiovasc Res 2006; 69:899-907.

This paper evidences the relation between altered cardiac PPAR expression and increased apoptosis in failing human hearts of hypertensive origin.

6 Gonzalez A, López B, Diez J. New directions in the assessment and treatment of hypertensive heart disease. Curr Opin Nephrol Hypertens 2005; 14:428434.

7 van Empel VP, Bertrand AT, Hofstra L, et al. Myocyte apoptosis in heart failure.

- Cardiovasc Res 2005; 67:21-29.

This is a review of the apoptotic pathways operative in cardiac muscle, focusing on the therapeutic options in human heart failure.

8 Garg S, Narula J, Chandrashekhar Y. Apoptosis and heart failure: clinical -• relevance and therapeutic target. J Mol Cell Cardiol 2005; 38:73-79.

Excellent review focused on the clinical relevance of apoptosis in human and experimental heart failure.

9 Diez J, Gonzalez A, Lopez B, Querejeta R. Mechanisms of disease: pathologic

- structural remodeling is more than adaptive hypertrophy in hypertensive heart disease. Nat Clin Pract Cardiovasc Med 2005; 2:209-216.

This review focuses on the mechanisms of hypertensive ventricular fibrosis and its clinical consequences.
10 Díez J, Querejeta R, López B, González A, et al. Losartan-dependent regression of myocardial fibrosis is associated with reduction of left ventricular chamber stiffness in hypertensive patients. Circulation 2002; 105:2512-2517.

11 Olivetti G, Melissari M, Balbi T, et al. Myocyte cellular hypertrophy is responsible for ventricular remodelling in the hypertrophied heart of middle aged individuals in the absence of cardiac failure. Cardiovasc Res 1994; 28 : 1199-1208.

12 Hein S, Arnon E, Kostin S, et al. Progression from compensated hypertrophy to failure in the pressure-overloaded human heart: structural deterioration and compensatory mechanisms. Circulation 2003; 107:984-991.

13 Anversa P, Kajstura J, Olivetti P. Myocyte death in heart failure. Curr Opin Cardiol 1996; 11:245-251.

14 Bing OHL, Ngo HQ, Humphries DE, et al. Localization of $\alpha_{1}$ (I) collagen mRNA in myocardium from the spontaneously hypertensive rat during the transition from compensated hypertrophy to failure. J Mol Cell Cardiol 1997; 29:23352344.

15 Chandrashekhar $Y$. Role of apoptosis in ventricular remodeling. Curr Heart

- Fail Rep 2005; 2:18-22.

This review highlights the importance of apoptosis in the progression seen in patients with heart failure and emphasizes the pharmacologic or gene therapy modulation of the apoptotic process.

16 de las Fuentes L, Herrero P, Peterson LR, et al. Myocardial fatty acid metabolism. Independent predictor of left ventricular mass in hypertensive heart disease. Hypertension 2003; 41:83-87.

17 Beer M, Seyfarth T, Sandstede J, et al. Absolute concentrations of high-energy phosphate metabolites in normal, hypertrophied, and failing human myocardium measured noninvasively with ${ }^{31} \mathrm{P}$-SLOOP magnetic resonance spectroscopy. J Am Coll Cardiol 2002; 40:1267-1274.

18 Crow MT, Mani K, Nam YJ, Kitsis RN. The mitochondrial death pathway and cardiac myocyte apoptosis. Circ Res 2004; 95:957-970.

19 Li Z, Bing OH, Long $X$, et al. Increased cardiomyocyte apoptosis during the transition to heart failure in the spontaneously hypertensive rat. Am J Physiol 1997; 272:H2313-H2319.

20 Bishopric NH, Andreka P, Slepak T, Webster KA. Molecular mechanisms of apoptosis in the cardiac myocyte. Curr Opin Pharmacol 2001; 1:141-150.

21 Leri A, Kajstura J, Anversa P. Cardiac stem cells and mechanisms of myo-. cardial regeneration. Physiol Rev 2005; 85:1373-1416.

This review discusses current understanding of the role that progenitor cells may have in the regeneration of the diseased heart.

22 Nadal-Ginard B, Kajstura J, Anversa P, Leri A. A matter of life and death: cardiac myocyte apoptosis and regeneration. J Clin Invest 2003; 111:14571459.

23 Communal C, Sumandea M, de Tombe $P$, et al. Functional consequences of caspase activation in cardiac myocytes. Proc Natl Acad Sci USA 2002; 99: $6252-6256$.

24 Lancel S, Joulin O, Favory R, et al. Ventricular myocyte caspases are directly

- responsible for endotoxin-induced cardiac dysfunction. Circulation 2005; 111:2596-2604.

This paper outlines the relationship between caspase activation and reduced contractility reserve in an experimental model of rat treatment with endotoxin injection.

25 González A, Fortuño MA, Querejeta R, et al. Cardiomyocyte apoptosis in hypertensive cardiomyopathy. Cardiovasc Res 2003; 59:549-562.

26 Moncrief J, McGavigan AD, Dunn FG. Arrhythmias, sudden death and syncope in hypertensive cardiovascular disease. Card Electrophysiol Rev 2002; 6:36-41

27 Nerheim P, Krishnan SC, Olshansky B, Shivkumar K. Apoptosis in the genesis of cardiac rhythm disorders. Cardiol Clin 2001; 19:155-163.

28 Hakumaki JM, Brindle KM. Techniques: Visualizing apoptosis using nuclear magnetic resonance. Trends Pharmacol Sci 2003; 24:146-149.

29 Narula J, Kietselaer B, Hofstra L. Role of molecular imaging in defining and denying death. J Nucl Cardiol 2004; 11:349-357.

30 Verhoven B, Schlegel RA, Williamson P. Mechanisms of phosphatidylserine exposure, a phagocyte recognition signal, on apoptotic T lymphocytes. J Exp Med 1995; 182:1597-1601.

31 Swairjo MA, Concha NO, Kaetzel MA, et al. $\mathrm{Ca}^{2+}$-bridging mechanism and phospholipid head group recognition in the membrane-binding protein annexin V. Nat Struct Biol 1995; 2:968-974.

32 Kietselaer BL, Hofstra $L$, Dumont EA, et al. The role of labeled annexin A5 in imaging of programmed cell death. From animal to clinical imaging. $\mathrm{Q} J \mathrm{Jucl}$ Med 2003; 47:349-361.

33 Flotats A, Carrió I. Non-invasive in vivo imaging of myocardial apoptosis and necrosis. Eur J Nucl Med 2003; 30:615-630. 
34 Matsuda R, Kaneko N, Kikuchi M, et al. Clinical significance of measurement of plasma annexin $\mathrm{V}$ concentration of patients in the emergency room. Resuscitation 2003; 57:171-177.

35 Renz A, Burek C, Mier W, et al. Cytochrome c is rapidly extruded from apoptotic cells and detectable in serum of anticancer-drug treated tumor patients. Adv Exp Med Biol 2001; 495:331-334.

36 Reeve JL, Duffy AM, O'Brien T, Samali A. Don't lose heart - therapeutic value

-. of apoptosis prevention in the treatment of cardiovascular disease. J Cell Mol Med 2005; 9:609-622.

Excellent review summarizing the mechanisms underlying cardiac apoptosis and the mediators/regulators involved with focus on the potential value of these molecules as therapeutic targets.

37 Landmesser U, Drexler H. Chronic heart failure: an overview of conventional -. versus novel approaches. Nat Clin Pract Cardiovasc Med 2005; 2:628-638. In this review the authors propose that prevention of apoptosis and regeneration of cardiac muscle would represent a shift of the current paradigm to approach the treatment of chronic heart failure.

38 Fortuño MA, González A, Ravassa S, et al. Clinical implications of apoptosis in hypertensive heart disease. Am J Heart Circ Physiol 2003; 284:H495H1506.

39 Qin F, Patel R, Yan C, Liu W. NADPH oxidase is involved in angiotensin II-

- induced apoptosis in H9C2 cardiac muscle cells: Effects of apocynin. Free Radic Biol Med 2006; 40:236-246.

This paper demonstrates the cardioprotective effects of apocynin, an antioxidant that inhibits angiotensin II-induced apoptosis via NADPH activation.

40 Khan M, Varadharaj S, Shobha JC, et al. C-phycocyanin ameliorates doxor-

- ubicin-induced oxidative stress and apoptosis in adult rat cardiomyocytes. J Cardiovasc Pharmacol 2006; 47:9-20.

This paper confirms the antiapoptotic effects of antioxidants in cardiomyocytes.

41 Korantzopoulos $P$, Galaris D, Papaioannides D, Siogas $K$. The possible role of oxidative stress in heart failure and the potential of antioxidant intervention. Med Sci Monit 2003; 9:120-125.

42 Freudenberger RS, Schwarz RP Jr, Brown J, et al. Rationale, design and organization of an efficacy and safety study of oxypurinol added to standard therapy in patients with NYHA class III-IV congestive heart failure. Expert Opin Invest Drugs 2004; 13:1509-1516.

43 Minhas KM, Saraiva RM, Schuleri KH, et al. Xanthine oxidoreductase inhibition causes reverse remodeling in rats with dilated cardiomyopathy. Circ Res 2006; 98:271-279.

44 Landmesser U, Spiekermann S, Dikalov S, et al. Vascular oxidative stress and endothelial dysfunction in patients with chronic heart failure: role of xanthineoxidase and extracellular superoxide dismutase. Circulation 2002; 106 3073-3078.

45 Fischer U, Schulze-Osthoff K. New approaches and therapeutics targeting -. apoptosis in disease. Pharmacol Rev 2005; 57:187-215.

Extensive review on the recent progress of apoptosis-based therapies.

46 Abbate A, Scarpa S, Santini D, et al. Myocardial expression of survivin, an

- apoptosis inhibitor, in aging and heart failure. An experimental study in the spontaneously hypertensive rat. Int J Cardiol 2005; 26 Oct. [Epub ahead of print]

This study provides the experimental basis for the modulation of the survivin mediated antiapoptotic pathway to prevent pressure overload heart failure.

47 Brocheriou V, Hagege AA, Oubenaissa A, et al. Cardiac functional improvement by a human $\mathrm{Bcl}-2$ transgene in a mouse model of ischemia/reperfusion injury. J Gene Med 2000; 2:326-333.
48 Toth $A$, Jeffers JR, Nickson $\mathrm{P}$, et al. Targeted deletion of Puma attenuates - cardiomyocyte death and improves cardiac function during ischemia/reperfusion. Am J Physiol Heart Circ Physiol 2006; 6 Jan. [Epub ahead of print]

This study establishes Puma, a BH3-only member of the $\mathrm{Bcl}-2$ protein family, as an essential mediator of cardiac myocyte death upon ischemia/reperfusion injury and offers a therapeutic target to limit cell loss in ischemic heart diasease.

49 Li Y, Cohen R. Caspase inhibitors and myocardial apoptosis. Int Anesthesiol - $\quad$ Clin 2005; 43:77-89.

Review of caspase inhibitors as the new therapeutic targets in cardiovascular medicine.

50 Laugwitz KL, Moretti A, Weig HJ, et al. Blocking caspase-activated apoptosis improves contractility in failing myocardium. Hum Gene Ther 2001; 12 2051-2063.

51 Tsukamoto O, Minamino T, Okada K, et al. Depression of proteasome - activities during the progression of cardiac dysfunction in pressure-overloaded heart of mice. Biochem Biophys Res Commun 2006; 340:11251133.

This paper establishes for the first time a direct relationship between depression of proteasome activities and the cardiac dysfunction resulting from cardiomyocyte apoptosis in the pressure-overloaded heart.

52 Morissette MR, Rosenzweig A. Targeting survival signaling in heart failure. - Curr Opin Pharmacol 2005; 5:165-170.

This excellent review describes the contribution of apoptotic signaling not only to the loss of cardiomyocytes but also to dysfunction of the surviving cells.

53 López N, Diez J, Fortuño MA. Characterization of the protective effects of cardiotrophin-1 against non-ischemic death stimuli in adult cardiomyocytes. Cytokine 2005; 30:282-292.

54 Zolk O, Ng LL, O'Brien RJ, et al. Augmented expression of cardiotrophin-1 in failing human hearts is accompanied by diminished glycoprotein 130 receptor protein abundance. Circulation 2002; 106:1442-1446.

55 Saetrum Opgaard O, Wang PH. IGF-I is a matter of heart. Growth Horm IGF - Res 2005; 15:89-94.

This article reviews recent research findings and outlines potential therapeutic implications of IGF-I in heart failure.

56 Anker SD, Volterrani M, Pflaum CD, et al. Acquired growth hormone resistance in patients with chronic heart failure: implications for therapy with growth hormone. J Am Coll Cardiol 2001; 38:443-452.

57 Burniston JG, Saini A, Tan LB, Goldspink DF. Aldosterone induces myocyte apoptosis in the heart and skeletal muscles of rats in vivo. J Mol Cell Cardiol 2005; 39:395-399.

58 Paraskevaidis IA, Parissis JT, Th Kremastinos D. Anti-inflammatory and antiapoptotic effects of levosimendan in decompensated heart failure: a novel mechanism of drug-induced improvement in contractile performance of the failing heart. Curr Med Chem Cardiovasc Hematol Agents 2005; 3:243247.

59 Mital S, Liao JK. Statins and the myocardium. Semin Vasc Med 2004; 4:377384.

60 Silverberg DS, Wexler D, Blum M, et al. Erythropoietin in heart failure. Semin Nephrol 2005; 25:397-403.

61 D'Ascia C, Cittadini A, Monti MG, et al. Effects of biventricular pacing on - interstitial remodelling, tumor necrosis factor- $\alpha$ expression, and apoptotic death in failing human myocardium. Eur Heart J 2006; 27:201-206.

This clinical study provides the first evidence that chronic resynchronization therapy reduces cardiac apoptosis in patients with advanced chronic heart failure. 